\title{
Can beating between different dynamo modes explain multiple magnetic cycles in solar - type stars
}

\author{
R. Simoniello ${ }^{1, a}$, C. Karoff ${ }^{2,3}$, and T. S. Metcalfe ${ }^{4}$ \\ 1 Laboratoire AIM, CEA/DSM-CNRS , Sap, F-91191, Gif -Sur Yvette, France \\ 2 Department of Geoscience, Aarhus University, Hoegh-Gukdbergs Gade 2,DK-8000 Aarhus C, Den- \\ mark \\ 3 Stellar Astrophysics Centre, Department of Physics and Astronomy, Aarhus University, Ny Munkgade \\ 120, DK-8000 Aarhus C, Denmark \\ 4 Space Science Institute, Boulder CO 80301 USA
}

\begin{abstract}
Stellar magnetic activity can be characterized by a chaotic, multiple or single cycle behavior. Sometimes cyclic activity can be interrupted by a flat behavior. The mechanism that produce such a diverse behavior in stellar atmosphere is a matter of debate. We decided to address this issue by investigating the properties of a sample of 40 stars with high quality cycles, selected from the original data provided by the Mount Wilson Observatory. This sample contains stars with single and secondary cycles, whose secondary periods are longer or shorter than the primary cycle.
\end{abstract}

\section{Introduction}

Long term observations of solar type stars [1] has revealed a pattern of correlated changes between rotation rates and chromospheric activity. Young stars shows high rotation rates, high activity levels and chaotic behavior in their stellar magnetic activity. Stars of intermediate ages, shows instead moderate levels of activity and rotation rates, and sometimes smooth cycles. Finally stars as old as the Sun shows slow rotation rates, low activity levels and cycles interrupted by flat behavior. Cyclic behavior of stellar magnetic activity naturally occurs in dynamo. Multiple magnetic dynamo configurations of different symmetry types might enhance multiple cycle periods as well as magnetic changes due to one polarity induce periodic modulation of the primary cycle [6]. The role played by the different component of the magnetic dynamo configuration has been suggested recently as a possible mechanism to explain the presence of the quasi-biennial periodicity (QBP) in helioseismic and solar activity proxies [5]. We, therefore, wonder if this mechanism might be at the origin of the observed multiple magnetic cycles in some stars.

\section{Data Analysis}

\subsection{On the origin of multiple magnetic cycles}

Fig.1 shows the relationship between the gyro chronological age [2] and the rotation rate. The Sun is shown in yellow. The stars rotating slower are the older ones, while the ones rotating faster are the youngest. We also found three stars with secondary cycles having slower rotation rate (above 30 days). These stars are K inactive stars. Many of the stars showing secondary cycles are younger, while

\footnotetext{
a e-mail: rosaria.simoniello@cea.fr
}

This is an Open Access article distributed under the terms of the Creative Commons Attribution License 4.0, which permits unrestricted use, distribution, and reproduction in any medium, provided the original work is properly cited. 

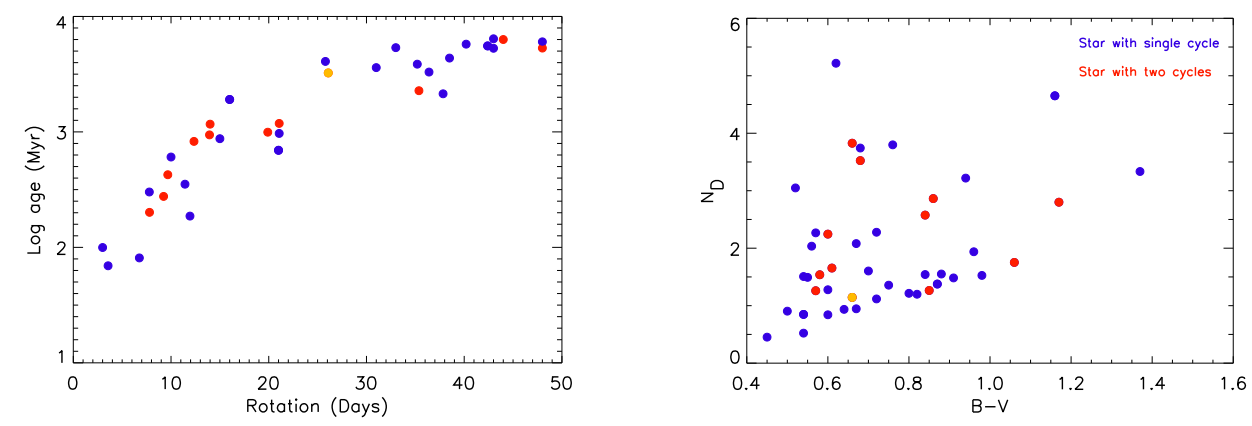

Fig. 1. Left panel: Star age as function of rotation rate. Right panel: Dynamo number as function of B-V.

the ones showing strict cycles span all range of ages. But there is a significant difference: while 50\% of stars showing single cycles are in the group of the oldest stars, only $20 \%$ of the stars showing secondary cycles are in the group of the oldest stars. In these stars a second dynamo mechanism acting in the subsurface layers might explain the origin of the secondary cycle [3], while for the younger stars, we should explore other possibilities.

\subsection{Dynamo efficiency}

Higher rotation rates also implies the excitation of more dynamo modes with different symmetry types. Younger stars, therefore, are expected to have a more efficient dynamo in this sense. If it is indeed the case, then we would expect that multiple cyclic behavior might be a function of the dynamo efficiency. Therefore we decided to investigate the relationship between the $\mathrm{N}_{D}$ (dynamo number) and $\mathrm{B}-\mathrm{V}$ as suggested by Durney (1983):

$$
N_{D}=5.2[(B-V)-0.44] \frac{\Omega}{\Omega_{0}}
$$

Fig. 3 shows that stars having multiple magnetic cycles mainly have a $\mathrm{N}_{D} \geq 1$. But if we take out the three slow rotator stars, then we would find that $\mathrm{N}_{D} \geq 1.5$

\section{Conclusion}

We have shown that stars showing secondary cycles mainly are younger than the ones showing only strict cycles. Furthermore, by plotting the dynamo number as function of B-V color, we have also found that stars showing secondary cycles seems to have slightly higher values of the $N_{D}$. This might therefore imply that what links the chaotic behavior of the young stars to the cyclic behavior of stars as old as the Sun is related to the efficiency and the capability of the dynamo to excite higher order multipoles. This is a preliminary work and still a lot of work needs to be done in order to be able to discern the different mechanism acting in the stars inducing multiple magnetic cycles.

\section{References}

1. Baliunas et al., ApJ 438, 269 (1995)

2. Barnes, S.A., ApJ 586, 464 (2003)

3. Böhm-Vitense, ApJ 657, 486 (2007)

4. Durney, M. and Robinson,R., PASP 93, 5 (1981)

5. Simoniello,R. et al., ApJ 657, 486 (2013)

6. Tobias, S. M., Weiss, N. O., \& Kirk, V., MNRAS 273, 1150 (1995) 